\title{
Checklist of Algerian fungi - Part 3: Laboulbeniales (Ascomycota)
}

\author{
Amrani $\mathbf{S}^{1 *}$ and Abdel-Azeem $\mathbf{A M}^{2}$ \\ 1* Laboratoire de Biologie des Sols, Faculté des Sciences Biologiques, Université des Sciences et de la Technologie \\ Houari Boumediène, BP 32 El Alia, Alger 16111, Algeria. \\ ${ }^{2}$ Laboratory of systematic Mycology, Botany Department, Faculty of Science, University of Suez Canal, Ismailia \\ 41522, Egypt.
}

Amrani S and Abdel-Azeem AM 2019 - Checklist of Algerian fungi - 3: Laboulbeniales (Laboulbeniomycetes, Ascomycota). Microbial Biosystems 4(1), 17- 30.

\begin{abstract}
Our third contribution to the checklist of Algerian fungi is dedicated to Labouls (Laboulbeniales, Laboulbeniomycetes) an a typical and very diversified group of microscopic fungi that unsure their nutrition and/or dispersal by being insect ectoparasites. By screening all available bibliographic sources and herbaria catalogues it was possible to delineate 66 species belonging to 26 genera that occurs in Algeria. Additionally a list of 8 erroneous or doubtful records unsupported by literature and/or herbarium material is provided. This checklist is the first comprehensive species list fully dedicated to Algerian Laboulbeniales.
\end{abstract}

Key words - Mycobiota, Laboulbeniales, biodiversity, literature and herbaria catalogs survey, species list.

\section{Introduction}

The Laboulbeniales is an order of fungi within the class Laboulbeniomycetes (Ascomycota) that consists of over 2000 species that are obligate ectoparasites of Arthropoda, mainly insects, but also mites and millipedes.

They are in most cases microscopical fungi $(0.04 \mathrm{~mm}$ to about $\mathrm{I} \mathrm{mm})$ that are unusual because they have no hyphae but cellular thalli, formed by enlargement and subsequent cell divisions of a two-celled ascospore. They parasitises the host through a foot cell which penetrates the insect cuticle with a haustorium and allows the fungus to extract nutrients from the insect body tissues. Therefore despite their parasitic habit, they cause usually little or no harm to the host, although they may impair host fitness if the level of infestation is high thus limiting their use as entomophagic agents.

First reports on Algerian laboulbeniales have been published by the famous American mycologist Roland Thaxter (1858-1932) after examination of Algerian insect specimens hold by some herbaria. In his five parts paper untitled "Contribution towards a monograph of the Laboulbeniaceae" (Thaxter 1895, 1908, 1901a, 1901b, 1902) he listed 3 genera and 9 species occurring in Algeria. Therefore the most comprehensive survey on Laboulbeniales of Algeria have been published by the French mycologist and botanist René Maire (1878-1949) who were established in the country and contributed drastically to the knowledge of it flora and mycbiota.

Submitted 9 April 2019, Accepted 25 June 2019, Published online 28 June 2019

Corresponding Author: Amrani S - e-mail - said_amrani@yahoo.com

This is an open access article distributed under the terms of the Creative Commons Attribution License, which permits unrestricted use, distribution, and reproduction in any medium, provided the original author and source are credited. 
He published 3 papers fully dedicated to the study of Laboulbeniales from North-Africa in which he raised the number of genera to 24 and the species to 57 from which two genera and 11 species where new to science (Maire 1912, 1916a, 1920).

In addition to the above-mentioned works at least the following ought to be mentioned as they contributed also significantly to the knowledge of Algerian laboulbeniales diversity Saccardo (1926), Picard (1913) and more recently Balazuc (1974, 1990), Santamaría et al. (1991). This later published a survey on the distribution of European Laboulbeniales in which he listed 62 species that occurs in Algeria. Therefore the reduced number and antiquity of the contributions on Laboulbeniales of Algeria lead one to believe that the country is clearly poorly explored and almost certainly contains many taxa of Laboulbeniales yet to be discovered. We trust that this will encourage scientists to investigate an important component of Algeria's biodiversity and to make it better known to the world's scientific community.

\section{Material and Methods}

\section{Study area}

Algeria, ca. 2.4 million $\mathrm{km}^{2}$ in area, is the largest country of Africa since the partition of Sudan in 2011. It is situated to the north of this continent, its entire northern coastline stretches for ca. $1200 \mathrm{~km}$ along the southern boundary of the Mediterranean Sea, while inland it is delimited clockwise, along a boundaries totalling ca. $6400 \mathrm{~km}$, by Tunisia, Libya, Niger, Mali, Mauritania, Sahrawi Arab Democratic Republic and Morocco. The country, which is mainly mountainous with an average altitude of $800 \mathrm{~m}$, stretches from north to south $\left(18^{\circ} 57^{\prime} \mathrm{N}\right.$ to $\left.37^{\circ} 08^{\prime} \mathrm{N}\right)$ to a distance of ca. $2000 \mathrm{~km}$ and from west to east $\left(08^{\circ} 39^{\prime} \mathrm{W}\right.$ to $\left.12^{\circ} 00^{\prime} \mathrm{E}\right)$ to a distance of ca. $2100 \mathrm{~km}$; the western parts of Algeria are only ca. $160 \mathrm{~km}$ from the Atlantic Ocean. The Mediterranean coastline and the two major mountain ranges, the Tell Atlas and the Saharan Atlas, delimitates southwards three major topographic and climatic regions (1) the Tell, (2) the Hauts-Plateaux and (3) the Sahara (Fig. 1).

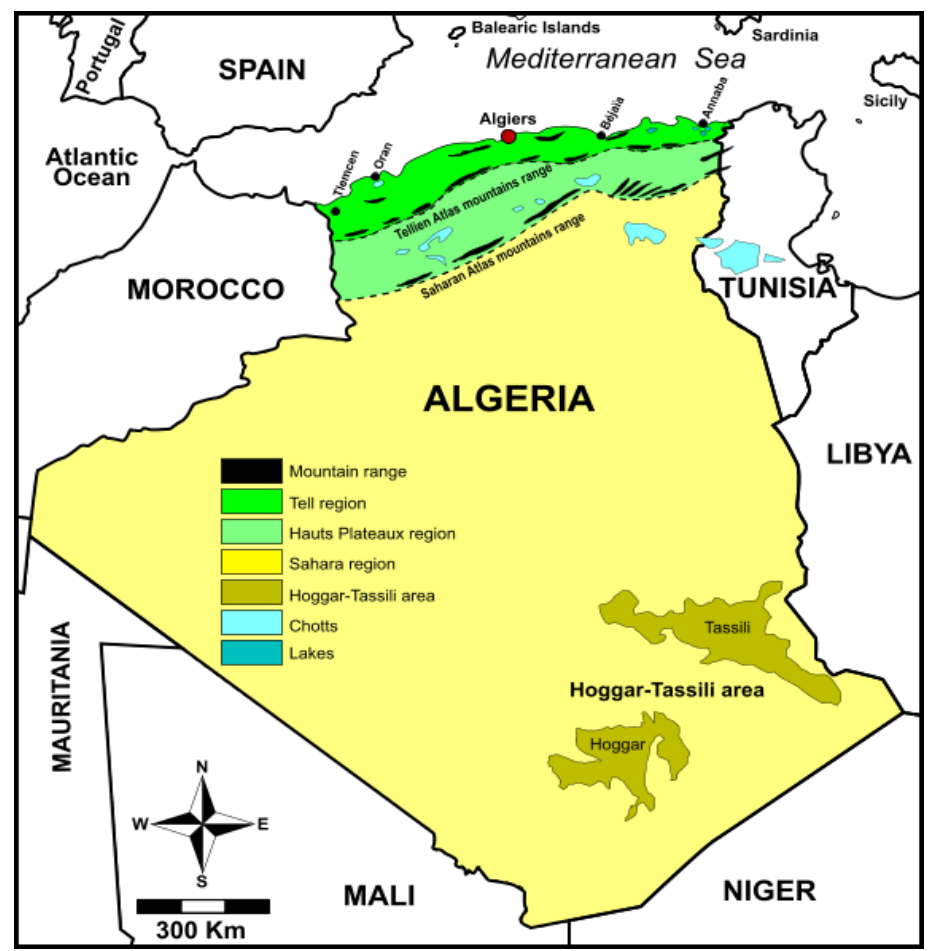

Fig 1 - Major biogeographical/bioclimatic regions of Algeria. 
The Tell region, stretching only 80-190 km inland, includes the coastal strip and the Tell Atlas mountains (Tellian Atlas), that consist of narrow coastal plains, hills and mountains. The annual precipitation ranges from semi-arid (400-600 $\mathrm{mm}$ ) to moderately humid with an increasing gradient from west to east where some permanent wetland areas occur. Although this region represents only ca. $4 \%$ of the Algerian territory, it includes $70 \%$ of the country's agricultural soils and hosts $65 \%$ of the population. The natural vegetation of this area is typically Mediterranean and many of the mountain slopes are covered with dense forest or scrub of mainly oaks (Quercus suber, Quercus ilex, Q. coccifera), junipers (Juniperus oxycedrus, J. thurifera J. phoenicea), aleppo pine (Pinus halepensis) and Atlas cedar (Cedrus atlantica).

The Hauts-Plateaux area is essentially a huge basin, ca. $950 \mathrm{~km}$ in length, lying at an altitude of ca. 1000 to $1400 \mathrm{~m}$ between the parallel mountains ranges of the Tell Atlas to the north and the Saharan Atlas to the south. This basin, which is $190 \mathrm{~km}$ at its widest point, includes a series of depressions running along a central south-west to north-east axis that support in some places shallow saline wetlands (Chotts and Sebkhas), which are seasonally flooded and become more saline as they gradually dry out. The region covers $13 \%$ of the country and hosts $26.5 \%$ of its population. The annual precipitation of the region is ca.150-400 $\mathrm{mm}$ and agriculture is limited to the cultivation of salt-tolerant cereals; natural vegetation is represented mainly by steppes of Stipa tenacissima, Artemisia herba-alba and Lygeum spartum.

The Saharan area is essentially a vast arid to desertic area characterized by very little rainfall $(<100 \mathrm{~mm}$ per annum) and only ephemeral streams. The Sahara desert is not homogenous and takes on many forms. In the far north-west it consists of stony and gravely desertic high land known as the Hamada du Draâ. Eastwards, the southern slopes of the Saharian Atlas descend over a distance of ca. $250 \mathrm{~km}$ to a central depression at an altitude of 200-500 m running south-west to north-east and filled by extensive ergs (vast sandy desertic areas with mobile dunes). In the centre of the region the land rises up to the stony plateau of Tademait, while further south it drops away into the Tidikelt depression that lies at an altitude of less than $200 \mathrm{~m}$. Southwards and eastwards of this depression, the land rises again through a series of mountains of different heights, including Mount Tahat (ca. $3000 \mathrm{~m}$ ), the highest peak in Algeria. This mountainous area, with its deep canyons and high plateaux, delimitate the Hoggar-Tassili region that can be considered as a fourth topographic and climatic region of Algeria due to its situation that make it under the influence of the west African monsoon.

The whole Saharan region covers $83 \%$ of the country, but hosts only $8.5 \%$ of its population in several oases developed from underground rivers or aquifers. Agriculture, in almost all cases limited to the oases, is based on the cultivation of the date palm and food crops. Natural vegetation cover is very scarce and represented mainly by highly adapted bushes and trees.

\section{Data Collection}

The species listed here were compiled from bibliographical and herbaria (fungaria) online databases sources. A main list of Algerian Laboulbeniales has been developed and the taxa are given in alphabetical sequence of taxa and accepted names are highlighted in bold. The names of authors of fungal taxa are abbreviated according to Kirk and Ansell (1992) and Kirk et al. (2008). Name corrections, authorities reported in this work were checked against the databases Index fungorum (www.indexfungorum.org) and Mycobank (www.mycobank.org). The insect host species for each taxon is provided by keeping the original name provided by the authors. 


\section{Checklist of Algerian Laboulbeniales.}

\section{Abbreviations used}

[Herbaria (Fungaria) Specimens]: Herbaria that hold specimens of the listed taxa are given between square brackets.

Cited herbaria (fungaria): M: Royal Botanical Garden of Madrid (Spain), NY: New York Botanical Garden (USA), PC: Muséum d'histoire Naturelle de Paris (France), SYRF: State University of New York Herbarium.

1. Amorphomyces italicus Speg.

Maire (1920), Santamaría et al. (1991), Santamaría (2000), Frank (2018a), Kedad and Bouznad (2018)

Trogophloeus (Carpelimus) halophilus (Staphylinidae).

2. Bordea coronata Maire (syn. Autophagomyces coronatus)

Maire (1916a), Saccardo (1926), Thaxter (1931), Benjamin (2000), Frank (2018a), Kedad and Bouznad (2018)

Brachygluta aubei (Staphylinidae).

3. Botryandromyces ornatus I.I. Tav. (syn. B. heteroceri, Misgomyces Heteroceri) Maire (1920), Petrak (1920), Balazuc (1973, 1990a), Tavares \& Majewski (1976), Santamaría et al. (1991), Weir (1994), Santamaría and Rossi (1999), Castaldo et al. (2004), Haelewaters et al. (2014), Rossi (2016), Rossi et al. (2019)

Heterocerus (Augiles) maritimus (Heteroceridae).

4. Cantharomyces bordei F. Picard

Maire (1912, 1916a), Picard (1912, 1913), Saccardo (1926), Thaxter (1931), Maire and Werner (1937), Santamaría et al. (1991), Benjamin (1989, 1992), Kedad and Bouznad (2018).

Limnichus sericeus (Limnichidae).

5. Cantharomyces numidicus Maire

Maire (1920), Petrak (1920), Saccardo (1926), Thaxter (1931), Huldén (1983),

Santamaría et al. (1991), Santamaría and Rossi (1999), Castaldo et al. (2004), Rossi et al. (2010), Frank (2018a), Kedad and Bouznad (2018)

Trogophloeus (Thinodromus) mannerheimi (Staphylinidae).

6. Cantharomyces orientalis Speg. (syn. C. abbreviatus)

Maire (1920), Petrak (1920), Saccardo (1926), Thaxter (1931), Siemaszko and

Siemaszko (1932), Huldén (1983), Santamaría et al. (1991), Majewski (1994), Castaldo et al. (2004), Rossi et al. (2010), Haelewaters and Yaakop (2014), Rossi (2016), Frank (2018a), Kedad and Bouznad (2018), Rossi and Bernardi (2018), Rossi et al. (2019) Trogophloeus corticinus (Staphylinidae).

7. Compsomyces lestevi Thaxt. (syn. C. lestevae)

Maire (1916a), Balazuc (1973, 1990a), Santamaría et al. (1991), Frank (2018a), Kedad and Bouznad (2018).

Lesteva fontinalis (Staphylinidae).

8. Corethromyces apotomi Thaxt. (syn. Eucorethromyces apotomi)

Maire (1916a), Balazuc (1990a), Santamaría et al. (1991), Kedad and Bouznad (2018). Apotomus flavescens (Carabidae). 
9. Corethromyces elegans Maire (syn. Peyerimhoffiella elegans)

Maire (1916a), Saccardo (1926), Thaxter (1931), Huldén (1983), Balazuc (1973),

Santamaría et al. (1991), Castaldo et al. (2004), Haelewaters et al. (2014), Frank (2018a)

Brachygluta reichei, B. dichroa (Staphylinidae).

10. Corylophomyces peyerimhoffii (Maire) R.K. Benj. (syn. Autophagomyces peyerimhoffii, Cryptandromyces peyerimhofii)

Maire (1920), Petrak (1920), Thaxter (1931), Benjamin (1970), Balazuc (1990a),

Santamaría et al. (1991)

Arthrolips obscurus var. sanctae-balmae (Corylophidae).

\section{Dimeromyces falcatus Paoli}

Balazuc (1974, 1990b), Santamaría et al. (1991)

Canestrinia carabicola (Canestriniidae).

12. Dimeromyces longitarsi Thaxt. (syn. D. bordei)

Maire (1920), Petrak (1920), Thaxter (1924), Saccardo (1926), Balazuc and Demaux

(1973), Santamaría et al. (1991), Kedad and Bouznad (2018)

Longitarsus parvulus (Chrysomelidae).

13. Dioicomyces anthici Thaxt. (syn. D. anthici var. fuscescens)

Maire (1916a, 1920), Petrak (1920), Saccardo (1926), Huldén (1983), Santamaría et al. (1991), Santamaría (2002), Kedad and Bouznad (2018)

Anthicus instabilis, A. rodriguesi (Anthicidae).

14. Euzodiomyces lathrobii Thaxt.

Maire (1916a), Balazuc (1973), Huldén (1983), Santamaría et al. (1991), Santamaría and Rossi (1999), Rossi et al. (2010), Frank (2018a)

Lathrobium anale (Staphylinidae).

15. Helodiomyces elegans F. Picard

Maire (1916a), Thaxter (1931), Balazuc (1973, 1990a), Huldén (1983), Santamaría et al. (1991), Rossi et al. (2010)

Dryops luridus (Dryopidae).

16. Herpomyces periplanetae Thaxt.

Maire (1916a), Santamaría et al. (1991)

Periplaneta americana (Blattidae).

17. Laboulbenia achenii Maire

Maire (1920), Petrak (1920), Saccardo (1926), Frank (2018)

Achenium tenellum (Staphylinidae).

18. Laboulbenia casnoniae Thaxt.

Maire (1916a, 1920), Santamaría et al. (1991)

Demetrias atricapilliis (Carabidae).

19. Laboulbenia clivinalis Thaxt.

Maire (1916a), Balazuc (1974, 1990a), Huldén (1983), Santamaría et al. (1991), Kedad and Bouznad (2018).

Clivina epsilon (Carabidae).

20. Laboulbenia deltomeri Maire

Maire (1916a), Fragoso (1924), Saccardo (1926), Siemaszko and Siemaszko (1932),

Maire and Werner (1937), Santamaría et al. (1991), Kedad and Bouznad (2018).

Deltomerus punctatissimus (Carabidae). 


\section{Laboulbenia dolicaontis Maire}

Maire (1920), Petrak (1920), Saccardo (1926), Frank (2018a).

Dolicaon densiventris (Staphylinidae).

22. Laboulbenia flagellata Peyr. (syn. L. flagellata var. bordei, L. flagellata var. romana, L. lepidoides var. bordei, L. algerina)

Maire (1912, 1916a, 1920), Picard (1913), Saccardo (1926), Balazuc (1974), Santamaría et al. (1991), Kedad and Bouznad (2018).

Harpalus tenebrosus (Carabidae), Platynus algirinus (Carabidae), Laemostenus fezzensis, Laemostenus prolixus (Carabidae), Olisthopus fuscatus (Carabidae).

23. Laboulbenia lagari Speg.

Spegazzini (1915), Maire (1916a), Saccardo (1926), Kedad and Bouznad (2018).

Platysma (Lagarus) ineptum (Carabidae)

Maire (1916a) stated that Spegazzini (1915) misidentified the host of this taxon since the subgenus Lagarus (genus Platysma) does not exist in North Africa and that the host reported by Spegazzini belongs to the subgenus Pedius an another subgenus of Platysma.

24. Laboulbenia luxurians Peyr.

Maire (1920), Balazuc (1974), Santamaría et al. (1991), Haelewaters et al. (2014).

Bembidion varium (Carabidae).

25. Laboulbenia macrotheca Thaxt. (syn. L. ophoni var. dilatata Maire)

Maire (1920), Petrak (1920), Santamaría et al. (1991).

Ophonus pubescens (Carabidae).

26. Laboulbenia nebriae Peyr. (syn. L. nebriae subsp. maghrebiana)

Thaxter (1908), Maire (1912, 1916a), Picard (1913), Balazuc (1974), Santamaría et al. (1991), Santamaría (1993), Kedad and Bouznad (2018).

Nebria rubicunda (Carabidae).

27. Laboulbenia ophoni Thaxt.

Thaxter (1899, 1908), Saccardo (1902), Maire (1912, 1916a, 1920), Picard

(1913),Fragoso (1924), Balazuc (1974, 1990a), Huldén (1983), Santamaría et al. (1991), Rossi et al. (2010), Kedad and Bouznad (2018).

Ophonus sp. (Carabidae), Scybalicus oblongiusculus (Carabidae).

28. Laboulbenia orthomi Thaxt.

Thaxter (1899, 1908), Saccardo (1902), Maire (1912, 1916a), Picard (1913), Kedad and

Bouznad (2018).

Orthomus aquila (Carabidae).

29. Laboulbenia pasqueti F. Picard

Maire (1916a), Balazuc (1974, 1990a), Santamaría et al. (1991), Santamaría (1993),

Kedad and Bouznad (2018).

Chlaeniellus olivieri (Carabidae).

30. Laboulbenia pedicellata Thaxt.

Maire (1916a, 1920), Balazuc (1974, 1990a), Santamaría et al. (1991), Frank (2018a),

Kedad and Bouznad (2018).

Reicheia lucifuga (Carabidae), Dyschirius punctatus (Carabidae), Aleochara tenuicornis (Staphylinidae). 
31. Laboulbenia polyphaga Thaxt.

Thaxter (1908), Maire (1912, 1916a, 1920), Picard (1913), Balazuc (1974), Santamaría et al. (1991), Kedad and Bouznad (2018).

Bradycellus puncticollis (Carabidae), Platyderus ruflcollis var. alacris (Carabidae), Acupalpus luteatus (Carabidae), Platyderus nolatus (Carabidae).

32. Laboulbenia proliferans Thaxt. (syn. L. proliferans var. interposita) Thaxter (1895, 1908), Maire (1912, 1916a, 1920), Picard (1913), Santamaría et al. (1991), Kedad and Bouznad (2018).

Chlaenius velutinus, C. aeratus, C. aeratus var. varvasi, Chlaenius sp. (Carabidae).

33. Laboulbenia rougetii Mont. \& C.P. Robin

Thaxter (1908), Maire (1912, 1916a, 1920), Picard (1913), Siemaszko and Siemaszko (1932), Santamaría et al. (1991), Kedad and Bouznad (2018).

Chlaenius aeneocephalus* C. chrysocephalus, C. fulgidicollis (Carabidae), Brachinus sclopeta, Brachinus sp. (Carabidae)

*This taxon have been reported for Algeria by Thaxter (1908) on specimens of Chlaenius aeneocephalus which not occurs in Algeria. Maire (1916a) stated that the right host is most probably Chlaenius chrysocephalus, a closely related species.

34. Laboulbenia siagonae F. Picard

Picard (1913), Maire (1916a), Saccardo (1926), Santamaría et al. (1991), Kedad and Bouznad (2018).

Siagona rufipes (Carabidae).

35. Laboulbenia slackensis Cépède and F. Picard

Maire (1920), Balazuc (1974, 1990a), Santamaría et al. (1991).

Pogonus chalceus (Carabidae).

36. Laboulbenia subterranea Thaxt.

Maire (1916a), Balazuc (1974), Santamaría et al. (1991), Kedad and Bouznad (2018).

Trechus maculipennis, T. rufulus, T. tingitanus (Carabidae).

37. Laboulbenia thaxteri Cépède \& F. Picard

Maire (1920), Santamaría et al. (1991), Weir (1994), Rossi et al. (2019).

Asaphidion (Tachypus) flavipes (Carabidae).

38. Laboulbenia vulgaris Peyr. (syn. L. abyssalis, L. lepesmei)

Maire (1945, 1916a, 1920), Saccardo (1926), Santamaría et al. (1991), Kedad and

Bouznad (2018).

Bembidium maculatum, B. minimum, B. dalmatinum subsp. africanum (Carabidae), Ocys (Oreocys) bedeli, Ocys (Oreocys) rufescens (Carabidae).

39. Mimeomyces speluncalis (Maire) I.I. Tav. (syn. Corethromyces speluncalis,

Sphaleromyces speluncalis)

Maire (1916a, 1920), Petrak (1920), Saccardo (1926), Thaxter (1931), Lepesme (1941), Frank (2018a).

Heterothops nigra (Staphylinidae).

40. Misgomyces dyschirii Thaxt.

Maire (1916a, 1920), Thaxter (1931), Balazuc (1973), Huldén (1983) Santamaría et al.

(1991) Rossi et al. (2010).

Dyschirius rufoaeneus (Carabidae).

41. Misgomyces reicheiae (Santam.) Santam.

Santamaría (1989c), Balazuc (1990a), Santamaría et al. (1991), [M]

Reicheia brisouti, R. kabyliana ssp. quezeli, R. subterranea (Carabidae). 
42. Monoicomyces homalotae Thaxt. (syn. M. homalotae var. geostibae) Maire (1916a, 1920), Saccardo (1926), Thaxter (1931), Balazuc (1974), Huldén (1983), Santamaría et al. (1991), Frank (2018a).

Atheta pertyi, A. amicula, A. parens (Staphylinidae), Geostiba peyerimhoffii (Staphylinidae).

43. Monoicomyces sanctae-helenae Thaxt.

Thaxter (1908, 1931), Maire (1912, 1916a), Picard (1913), Balazuc (1974), Huldén (1983), Santamaría et al. (1991), Frank (2018a), Kedad and Bouznad (2018). Oxytelus piceus*

* Thaxter (1908) cites as host insect for this taxon Oxytelus Iuteipennis (Staphylinidae) that which not occurs in Algeria. Maire (1916a) and Frank (2018) considers that it is most probably Oxytelus piceus (Staphylinidae) wich is a very similar species.

44. Peyerimhoffiella elegans Maire (syn. Corethromyces brachyglutae, C. elegans, Cryptandromyces elegans)

Maire (1916a), Saccardo (1926), Siemaszko and Siemaszko (1932), Balazuc(1973), Huldén (1983), Santamaría et al. (1991), Rossi et al. (2013), Haelewaters et al. (2014), Frank (2018a), Kedad and Bouznad (2018).

Brachygluta reichei, B. dichroa (Staphylinidae).

45. Peyritschiella furcifera (Thaxt.) I.I. Tav. (syn. Dichomyces furciferus, D. furcifer) Maire (1916a), Thaxter (1931), Santamaría et al. (1991), Frank (2018a), Kedad and Bouznad (2018).

Philonthus discoideus (Staphylinidae).

46. Peyritschiella princeps (Thaxt.) I.I. Tav. (syn. Dichomyces princeps) Maire (1916a), Thaxter (1931), Santamaría et al. (1991), Frank (2018a). Philonthus sordidus (Staphylinidae).

47. Peyritschiella protea Thaxt.

Maire (1920), Siemaszko and Siemaszko (1932), Balazuc (1974, 1990a), Huldén (1983), Santamaría et al. (1991), Haelewaters et al. (2017), Frank (2018a), Kedad and Bouznad (2018), Rossi et al. (2019).

Planeustomus miles (Staphylinidae).

48. Peyritschiella vulgata (Thaxt.) I.I. Tav. (syn. Dichomyces vulgatus)

Maire (1916a), Santamaría et al. (1991), Frank (2018a).

Philonthus minutes (Staphylinidae).

49. Rhachomyces fagniezi $\mathrm{F}$. Picard

Maire (1920), Thaxter (1931), Santamaría et al. (1991).

Zuphium baeticum (Carabidae).

50. Rhachomyces furcatus (Thaxt.) Thaxt.

Maire (1916a, 1920), Balazuc (1973), Huldén (1983), Santamaría et al. (1991), Frank

(2018a), Kedad and Bouznad (2018), Rossi et al. (2019).

Othius pilifer, O. panctulatus (Staphylinidae).

51. Rhachomyces lavagnei (F. Picard) W. Rossi (syn. Dimeromyces lavagnei)

Maire (1916a, 1920), Thaxter (1924), Saccardo (1926), Balazuc (1974), Santamaría et al. (1991), Rossi (2016), Kedad and Bouznad (2018).

Dyschirius sp. (Carabidae), Microlestes laevipennis (Carabidae). 
52. Rhachomyces peyerimhoffii Maire

Maire (1912, 1915, 1916a, 1916b), Picard (1913), Fragoso (1924), Saccardo (1926), Thaxter (1931), Santamaría et al. (1991), Kedad and Bouznad (2018), [M, NY, P, SYRF] Trechus (Trechopsis ) lapiei (Trechini).

53. Rhachomyces philonthinus Thaxt.

Maire (1920), Thaxter (1931), Huldén (1983), Santamaría et al. (1991).

Philonthus concinnus (Staphylinidae).

54. Rhachomyces stipitatus Thaxt. (syn. Rhachomyces stipitatus var. pallidus)

Maire (1912, 1916a), Picard (1913), Saccardo (1926), Lepesme (1941), Balazuc (1970, 1973, 1990a), Rossi (1978), Santamaría et al. (1991), Rossi et Santamaría (2001), Kedad and Bouznad (2018), [P]

Duvalius (Aphaenops) iblis (Carabidae).

55. Rickia peyerimhoffii Maire

Maire (1920), Saccardo (1926), Thaxter (1926), Siemaszko and Siemaszko (1932),

Balazuc (1974), Huldén (1983), Santamaría et al. (1991), Castaldo et al. (2004), Frank (2018), Kedad and Bouznad (2018), Rossi et al. (2019).

Scaphosoma agaricinum, Scaphosoma flavonotatum (Staphylinidae).

56. Scalenomyces endogaeus (F. Picard) I.I. Tav. (syn. Laboulbenia endogaea)

Balazuc (1971, 1974, 1990a), Santamaría et al. (1991), [P]

Reicheia sp.

57. Sphaleromyces obtusus Thaxt. (syn. Corethromyces obtusus)

Thaxter (1900, 1908, 1931), Saccardo (1902), Maire (1912, 1916a, 1920), Picard (1913),

Siemaszko and Siemaszko (1932), Santamaría et al. (1991), Frank (2018a), Kedad and

Bouznad (2018).

Lathrobium Illyricum*, Dolicaon illyricus

* Thaxter (1900) cites as host insect for this taxon Lathrobium Illyricum (Staphylinidae) that does not occur in Algeria. Maire (1916a) considers that it is most probably Dolicaon illyricus (Staphylinidae) wich is a very similar species.

58. Sphaleromyces propinquus Thaxt. (syn. Corethromyces propinquus)

Maire (1916a, 1920), Thaxter (1931), Santamaría et al. (1991), Frank (2018a), Kedad and Bouznad (2018).

Dolicaon illyricus (Staphylinidae).

59. Stichomyces conosomatis Thaxt.

Maire (1920), Thaxter (1931), Santamaría et al. (1991), Castaldo et al. (2004),

Haelewaters et al. (2017), Rossi et al. (2019).

Conosoma testaceum (Staphylinidae).

60. Stigmatomyces crassicollis Thaxt. (syn. Stigmatomyces papuanus var. leiostoma)

Maire (1920), Saccardo (1926), Thaxter (1931), Santamaría et al. (1991), Rossi et al. (2013).

Leptocera (Limosina) fontinalis (Limosininae).

61. Stigmatomyces micrandrus Thaxt.

Rossi (1988), Santamaría et al. (1991).

Psilopa nana (Ephydridae).

62. Stigmatomyces purpureus Thaxt.

Rossi (1988, 2016), Rossi et al. (2013)

Scatella stagnalis (Ephydridae). 
63. Stigmatomyces rugosus Thaxt.

Rossi (1988), Santamaría et al. (1991), Rossi et al. (2013).

Psilopa flavipalpis (Ephydridae).

64. Stigmatomyces trianguliapicalis T. Majewski

Rossi (1988), Santamaría et al. (1991).

Parydra hecate (Ephydridae).

65. Teratomyces actobii Thaxt. (syn. Teratomyces atropurpureus)

Maire (1920), Petrak (1920), Benjamin (1968), Balazuc (1974, 1990a), Santamaría et al. (1991), Rossi et al. (2019).

Actobius signaticornis (Staphylinidae).

66. Triceromyces hydrometrae R.K. Benj.

Santamaría and Rossi (1999), Santamaría (2008).

Hydrometra stagnorum (Hydrometridae).

Erroneous or doubtful records weakly supported by literature and herbarium material.

1. Euzodiomyces capillarius Cépède \& F. Picard

Frank (2018a).

Lobrathium anale

This taxon is quoted for Algeria only by Frank (2018) probably by error since the given references (Maire 1916a, Balazuc 1973b) and host insect (Lobrathium anale) are the same to those supplied by this author for Euzodiomyces lathrobi (Staphylinidae).

2. Laboulbenia acupalpi Speg.

Santamaría (1989b), Balazuc (1990a), Santamaría et al. (1991), Haelewaters et al. (2015).

Acupalpus sp.

Santamaría (1989b) consider possible the occurence of this taxon in Algeria on the basis of Thaxter's report (1908) on the presence of Laboulbenia polyphaga on Acupalpus luteatus ((as Bradycellus puncticollis (Carabidae) and included it later in the list of Laboulbeniales that occurs in Algeria (Santamaría et al. 1991).

3. Laboulbenia egens Speg.

Balazuc (1990a), Santamaría et al. (1991).

Tachys (Tachyura) parvula var. curvimanus (Carabidae)

Santamaría et al (1991) consider that this taxon occurs in Algeria after examination of an illustration of an Algerian specimen from Balazuc's collection classified as Laboulbenia tachyis but having all the characteristics of L. egens.

4. Laboulbenia gyrinicola Speg.

Maire (1916a), Siemaszko and Siemaszko (1932), Balazuc (1971b), Huldén 1983

Santamaría et al. (1991).

Gyrinus urinator, G. natator (Gyrinidae)

Reported by several authors as present in Algeria only on the basis of Maire (1916a) reports and in which the localities mentioned (Tanger, Aïn-Hammera = Aïn Hamra) are in fact located in Morocco. 


\section{Laboulbenia polyhirmae Thaxt.}

Thaxter (1899), Saccardo (1902), Thaxter (1908), Picard (1913), Kedad and Bouznad (2018).

Polyhirma sp. (Carabidae)

Reported by several authors as present in Algeria only on the basis of Thaxter (1899) reports, this taxon must be excluded from the list of Algarian Laboulbeniales since the host cited by Thaxter (Polyhirma sp.) is a genus that lack totally in North Africa (Maire 1916a). and furthermore the locality mentioned by the author (Tangar, Algeria) correspond in all likelihood to Tanger in Morocco.

6. Laboulbenia tachyis Thaxt. Maire (1920), Barragán et al. (2013).

Tachys haemorrhoidalis var. socius(Carabidae)

Barragán et al. (2013) Consider that all the records of Laboulbenia tachyis reported on various species of "Tachys" sensu lato from outside the American continent should be considered very carefully. Furthermore Santamaría et al (1991) stated that report of this species by Maire (1920) for Algeria is possibly erraneous.

7. Monoicomyces invisibilis Thaxt. (syn. Eumonoicomyces californicus, Monoicomyces californicus)

Maire (1920), Thaxter (1931), Frank (2018a), Kedad and Bouznad (2018).

Oxytelus inustus (Staphylinidae)

Reported by Maire (1920) from Algeria, this taxon was considered soon by Thaxter (1931) on the basis of the illustration of the antheridium given by Maire (1920) of some other fungal species than Monoicomyces californicus.

\section{Conflict of Interest}

The authors do not have any conflicts of interest.

\section{Acknowledgements}

The authors would like to thank the two anonymous reviewers for the valuable suggestions.

\section{References}

Balazuc J. 1970. Commentaires sur les Rhachomyces parasites des Trechidae, avec descriptions de formes nouvelles (Ascomycètes, Laboulbéniales). Ann. Soc. Ent. Fr., 6 (3): 677-699.

Balazuc J. 1971a. Laboulbéniales inédites, parasites de Carabiques et d'un catopide. Nouv. Rev. Entomol., 1(1): 107-117.

Balazuc J. 1971b. Notes sur les Laboulbéniales: II. Laboulbenia parasites des Gyrinus (plus particulièrement européens et nord-africains). Bulletin mensuel de la Société Linnéenne de Lyon, 40 (6):160-168. DOI 10.3406/linly.1971.9925.

Balazuc J. 1973. Laboulbéniales de France. Bulletin mensuel de la Société Linnéenne de Lyon, 42: 244-256, 280-285.

Balazuc J. 1974. Laboulbéniales de France (suite). Bulletin mensuel de la Société Linnéenne de Lyon, 43: 12-21, 57-64, 73-79, 253-262, 295-315, 346-368.

Balazuc J. 1990a. Catalogue actuel des Laboulbéniales (Ascomycètes parasites) de la France métropolitaine. L'entomologiste, 46(5): 219-232. 
Balazuc J. 1990b. les laboulbéniales (ascomycètes) parasites d'acariens description d'une espèce nouvelle. Acarologia, 31 (2): 103-112.

Balazuc J., Demaux J. 1973. Une nouvelle espèce de Laboulbenia (Ascomycètes, Laboulbeniales), parasite de Coléoptères Chrysomélides Hispinae. Bulletin mensuel de la Société linnéenne de Lyon, 42 (special issue, december 1973), 7-9. DOI: 10.3406/linly.1973.10026.

Barragán A., Bernardi M. and Rossi W. 2013. New records of Laboulbenia (Fungi, Ascomycota) from Ecuador and other countries, Webbia, 68 (1): 25-34. DOI:10.1080/00837792.2013.779816.

Benjamin R.K. 1968. Sandersoniomyces, A New Genus of Laboulbeniales Allied to Diplomyces, Symplectromyces and Teratomyces. Aliso, 6 (4):1-16.

Benjamin R.K. 1970. Laboulbeniales on Semiaquatic Hemiptera. II. Autophagomyces, Dioicomyces, and Prolixandromyces Gen. Nov. Aliso, 7 (2): 165-182.

Benjamin R.K. 1989. Taxonomy and morphology of Aporomyces (Laboulbeniales). Aliso 1 (2):335-367.

Benjamin R.K. 1992. A new genus of Laboulbeniales (Ascomycetes) on a species of Phalacrichus (Coleoptera: Dryopoidea; Limnichidae), with a note on mirror-image asymmetry in the order. Aliso, 13 (3): 427-446.

Benjamin R.K. 2000. Autophagomyces, Bordea, and a New Genus, Rossiomyces, (Laboulbeniales), Aliso, 19 (2): 99-136.

Castaldo D., Rossi W. and Sabatini F. 2004. Contribution to the knowledge of the Laboulbeniales from Greece. Plant Biosystems, 138 (3): 261-269.

Fragoso R.G. 1924. Acerca de algunos Laboulbeniales de España y de Marruecos. Boletín de la Real Sociedad Española de Historia Natural, 24: 405-414.

Frank J.H. 2018. A Worldwide Checklist of Parasites of Staphylinidae. In O. Betz, J. Klimaszewski, U. Irmler (eds.), Biology of Rove Beetles (Staphylinidae), Springer, Cham, Switzerland, pp. 183-224. DOI 10.1007/978-3-319-70257-5_10.

Haelewaters D, de Kock G and van Wielink P. 2015. Nieuwe Laboulbeniales in De Kaaistoep. In: Peeters T, van Eck A, Cramer T (eds.) Natuurstudie in De Kaaistoep. Verslag 2014, 20e onderzoeksjaar. TWM Gronden BV, Natuurmuseum Brabant \& KNNV-afdeling Tilburg, pp. 11-18.

Haelewaters D., Verhaeghen S.J.C., Ríos Gonzáles, T. A., Bernal Vega J.A. and Villarreal Saucedo R.V. 2017. New and interesting Laboulbeniales from Panama and neighboring areas. Nova Hedwigia, 105 (3-4): 267-299. DOI 10.1127/nova_hedwigia/2017/0410.

Haelewaters D., Vorst O. and de Kesel A. 2014. New and interesting Laboulbeniales (Fungi, Ascomycota) from the Netherlands. Nova Hedwigia, 98 (1-2): 113-125. DOI: 10.1127/0029-5035/2013/0150.

Haelewaters D. and Yaakop S. 2014. New and interesting Laboulbeniales from southern and southeastern Asia. Mycotaxon, 129 (2): 439-454.

Huldén L. 1983. Laboulbeniales (Ascomycetes) of Finland and adjacent parts of the U.S.S.R. Karstenia, 23: 31-136.

Lepesme P. 1941. Catalogue des Laboulbéniales de la Collection François Picard. Bulletin du Museum National d'Histoire Naturelle, 8 (1): 481-488.

Maire R. 1912. Contribution à l'étude des Laboulbéniales de l'Afrique du Nord. Bulletin de la Société d'Histoire Naturelle de l'Afrique du Nord, 4 (9): 194-199.

Maire R. 1916a. Deuxième contribution à l'étude des Laboulbéniales de l'Afrique du Nord. Bulletin de la Société d'Histoire Naturelle de l'Afrique du Nord, 7 (1): 6-39.

Maire R. 1916b. Sur une nouvelle Laboulbéniale parasite des Scaphidiidae. Bull. Sci. Fr. Belg., 49 (7): 290-296. 
Maire R. 1920. Troisième contribution à l'etude des Laboulbéniales de l'Afrique du Nord.Bulletin de la Société d'Histoire Naturelle de l'Afrique du Nord, 41: 123-138, 143-158, 159-170.

Maire R. 1945. Etudes mycologiques - Fascicule 5. Bulletin de la Société d'Histoire Naturelle de l'Afrique du Nord, 36(3): 24-42.

Maire R. 1915. Schedae ad Mycothecam boreali-Africanam. Bulletin de la Société d'Histoire Naturelle de l'Afrique du Nord, 7: 66-68, 79-84, 127-134, 139-156.

Maire R.C.J.E. and Werner R.G. 1937. Fungi Maroccani. Mémoires de la Société des Sciences Naturelles du Maroc, 45: 1-147.

Majewski T. 1994. The Laboulbeniales of Poland. Polish Botanical Studies, 7:1-466.

Petrak F. 1920. Mykologische Notizen. I. Annales Mycologici 17 (2-6): 59-100.

Picard F. 1912. Description de deux Laboulbéniacées nouvelles, parasites de Coléoptères. Bulletin de la Société Entomologique de France, 17 (8): 178-181.

Picard F. 1913. Contribution a l'étude des laboulbéniacées d'Europe et du nord de l'Afrique. Bulletin Trimestriel de la Société Mycologique de France, 29 (1): 503-571,.

Kekad A. and Bouznad Z. 2018. Cayalogie des champignons d'Algérie. Tarzalt M. Arts graphiques Edition, Alger-Algeria, 334 pages.

Rossi W. 1978. Sui Rhachomyces (Ascomycetes, Laboulbeniales) parassiti dei Duvalius italiani (Coleoptera, Carabidae,Trechini). Int. J. Speleol., 10: 323 - 330.

Rossi W. 1988. New or interesting Laboulbeniales (Ascomycetes) parasitic on Diptera, Webbia 42(2): 171-178, DOI: 10.1080/00837792.1988.10670436.

Rossi W. 2016. Contribution to the knowledge of the Laboulbeniales (Ascomycota) from Turkey. Webbia, 71(2): 265-276. DOI: 10.1080/00837792.2016.1222668.

Rossi W. and Bernardi M. 2018. New species and new records of Laboulbeniales (Ascomycota) from Thailand. Mycosphere 9(6): 1151-1172. DOI 10.5943/mycosphere/9/6/6.

Rossi W., Guéorguiev B., Georgiev G. and Stoianova D. 2019. Laboulbeniales (Ascomycota) from Bulgaria and other countries, Plant Biosystems. 153 (1): 48-59. DOI: 10.1080/11263504.2018.1454531.

Rossi W., Máca J. and Vávra J. 2010. New records of Laboulbeniales (ascomycota) from the Czech Republic and Slovakia. Polish Botanical Journal, 55(2): 343-351.

Rossi W. and Santamaría S. 2001. Laboulbeniales (Ascomycetes) in the Mediterranean area. Bocconea 13: 125-130.

Rossi W., Santamaría S. and Andrade R. 2013. Notes on the Laboulbeniales (Ascomycota) parasitic on Diptera from Portugal and other countries, Plant Biosystems, 147(3): 730742. DOI: $10.1080 / 11263504.2012 .753132$.

Saccardo P. A. 1902. Sylloge fungorum omnium hucusque cognitorum, Vol. XVI - Pars V. Padova \& Berlin.

Saccardo P. A. 1926. Sylloge fungorum omnium hucusque cognitorum, Vol. XXIV - Pars X. Padova \& Berlin.

Santamaría S. 1989c. Two new species of Laboulbenia (Laboulbeniales ) parasitic on endogean carabid beetles. Mycotaxon 35(1): 163-168.

Santamaría S. 1989a. Contribución al conocimiento de los Laboulbeniales (Fungi, Ascomycotina) ibéricos, I11. Orsis, 8: 21-31.

Santamaría S. 1989b. El orden Laboulbeniale s (Fungi . Ascomycotina ) en la Península Ibérica e Islas Baleares. Edicions especiáis de la Societat Catalana de Micologia. 3, 396 pages.

Santamaría S. 1993. Contribución al conocimiento de los laboulbeniales (Ascomycotina) ibéricos, IV. Género Laboulbenia: adiciones y correcciones. Revista Catalana Micologia, 19: 5-13.

Santamaría S. 2000. Taxonomy and morphology of Amorphomyces (Laboulbeniales). Mycol. Res., 104 (11):1389-1398. 
Santamaría S. 2002. A taxonomic revision of the genus Dioicomyces (Laboulbeniales). Mycol. Res. 106 (5): 615-638. DOI: 10.1017\}S0953756202005816.

Santamaría S., Balazuc J. and Tavares, I. I. 1991. Distribution of the European Laboulbeniales (Fungi, Ascomycotina). An annotated list of species. Treballs de l'Institut Botanic de Barcelona, 14: 1-123.

Santamaría S. and Rossi W. 1999. New or interesting laboulbeniales (ascomycota) from the Mediterranean region, Plant Biosystems, 133 (2): 163-171. DOI: 10.1080/11263509909381545.

Siemaszko J. and Siemaszko W. 1932. Owadorosty polskie i palearktyczne, II Polskie Pismo Entomologiczne, 10: 149-188.

Tavares I.I. and Majewski T. 1975. Siemaszkoa and Botryandromyces, two segregates of misgomyces (Laboulbeniales). Mycotaxon, 3 (2): 193-208.

Spegazzini C. 1915. Laboulbeniali ritrovate nelle collezioni di alcuni musei italiani. Anales del Museo Nacional de Historia Natural Buenos Aires, 26: 451-511.

Tahxter R. 1899. Preliminary Diagnoses of New Species of Laboulbeniaceæ. I. Proceedings of the American Academy of Arts and Sciences, 35 (9): 153-209.

Thaxter R. 1895. Contribution towards a Monograph of the Laboulbeniaceæ. Memoirs of the American Academy of Arts and Sciences, 12 (3): 189, 429.

Thaxter R. 1908. Contribution towards a Monograph of the Laboulbeniaceæ: Part II. Memoirs of the American Academy of Arts and Sciences, 13 (6): 219, 469.

Thaxter R. 1924. Contribution towards a Monograph of the Laboulbeniaceæ.: Part III. Memoirs of the American Academy of Arts and Sciences, 14 (5): 313-409, 411-426.

Thaxter R. 1926. Contribution towards a Monograph of the Laboulbeniaceæ: Part. IV. Memoirs of the American Academy of Arts and Sciences, 15 (4) 1926): 431-555, 557-580.

Thaxter R. 1931. Contribution towards a monograph of the Laboulbeniaceae. Part V. Memoirs of the American Academy of Arts and Sciences, 16 (1): 1-435.

Weir A. 1994. Further records of Laboulbeniales from collections of British Coleoptera. Mycol. Res., 98: 433-444. 